\title{
Incomplete financial markets and jumps in asset prices*
}

\author{
Hervé Crès $^{\dagger} \quad$ Tobias Markeprand ${ }^{\ddagger} \quad$ Mich Tvede $^{\S}$
}

\begin{abstract}
For incomplete financial markets jumps in both prices and consumption can be unavoidable. We consider pure-exchange economies with infinite horizon, discrete time, uncertainty with a continuum of possible shocks at every date. The evolution of shocks follows a Markov process and fundamentals depend continuously on shocks. It is shown: (1) equilibria exist; (2) for effectively complete financial markets asset prices depend continuously on shocks; and, (3) for incomplete financial markets there is an open set of economies $\mathscr{U}$ such that for every equilibrium of every economy in $\mathscr{U}$, asset prices at every date depend discontinuously on the shock at that date.
\end{abstract}

Keywords financial markets $\cdot$ general equilibrium $\cdot$ jumps in asset prices

JEL-classification D52 $\cdot$ D53 $\cdot$ E32 $\cdot$ G11 $\cdot$ G12

${ }^{*}$ The authors wish to thank an anonymous referee for constructive suggestions and M. Pascoa as well as participants in GEdays in York for interesting comments.

${ }^{\dagger}$ New York University in Abu Dhabi, PO Box 129188 Abu Dhabi, United Arab Emirates, email: herve.cres@nyu.edu

$\ddagger$ DREAM, Amaliegade 44, 1256 Copenhagen K, Denmark, email: tma@ dreammodel.dk

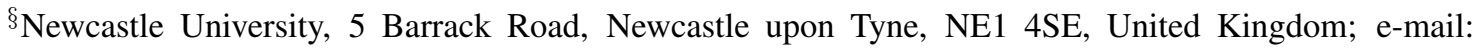
mich.tvede@ncl.ac.uk. 


\section{Introduction}

Consider an economy with uncertainty. Suppose fundamentals of the economy depend continuously on the state of the world, while asset prices depend discontinuously on the state of the world, or in other words asset prices jump. Then markets amplify uncertainty in the sense that on top of the uncertainty about fundamentals there is uncertainty about whether asset prices jump or not. The present paper shows that the amplification of uncertainty can be unavoidable by providing a general equilibrium explanation of jumps in asset prices.

The study of jumps in asset returns has a long history in finance. Indeed, the consequences of jumps have been considered since Merton (1976). Empirically jumps have received some attention in recent years as can be seen in Andersen, Benzoni \& Lund (2002), Christoffersen, Jacobs \& Ornthanalai (2011) and Eraker, Johannes \& Polson (2003). Estimates in these articles range from 1-2 to 8-9 jumps per year and jumps accounting for from 8-15 to 12-15 percent of the total variance for the S\&P 500.

The theoretical literature on jumps in asset prices is limited. A couple of contributions use partial equilibrium models. In Balduzzi, Foresi \& Hait (1997) consider a model with a mix of utility maximizing investors and buy-and-hold investors. Buy-and-hold investors trade only in case prices hit lower or upper barriers, perhaps because of transaction costs. It is shown that, if all buy-and-hold investors have identical barriers, then prices jump in case prices hit the barriers. Lim, Martin \& Teo (1998) consider a partial disequilibrium model with continuous time. Price movements depend on the difference between demand and supply. Some suppliers use portfolio-insurance hedging strategies, which result in supply being downward sloping for low prices. Therefore there can be multiple equilibria and the movement of prices depend on the views of agents. It is shown that in case agents switch views asset prices jump.

A couple of contributions study jumps in asset prices in general equilibrium models with infinitely lived consumers. Bansal \& Shaliastovich (2009) consider pure-exchange economies with discrete time, uncertainty and incomplete information. There is a representative agent, who can buy new information. It is shown that prices jump in case the representative agent buys new information. Calvet \& Fisher (2008) consider pure-exchange economies with infinite horizon, continuous time and uncertainty. Asset returns follow geometric Brownian motions with jumps in drift and volatility parameters. For these processes asset returns depend continuously on time, but asset prices depend discontinuously on time: jumps in the drift parameter result in jumps in the expected discounted dividends of assets; and, jumps in the volatility parameter result in jumps in the expected discounted utility of dividends of assets so asset prices jump.

An issue related to jumps is volatility. Price volatility is studied in Calvet (2001) and 
Citanna \& Schmedders (2005). The set of possible shocks at every date is finite and the evolution of shocks follows a general Markov process. The basic idea is that incomplete financial markets prevent consumption smoothing, so prices are more volatile with incomplete financial markets than with complete financial markets. Consumption volatility for pure-exchange economies with discrete time, uncertainty and incomplete financial markets is considered in Beker \& Chattopadhyay (2010) where dynamic properties of equilibria are studied. For a large class of economies with one good and two consumers it is shown that in any equilibrium either consumption of one of the consumers is eventually zero or consumption of both consumers is arbitrarily close to zero at infinitely many dates.

Other papers have studied incomplete financial markets and asset prices in pure-exchange economies with infinite horizon, discrete time, uncertainty and incomplete financial markets. In Telmer (1993) and Lucas (1994) endowments of consumers are hit by transitory idiosyncratic shocks. In both papers it is found that consumers are able to smooth consumption by accumulating assets. In Constantinides \& Duffie (1996) endowments of consumers are hit by persistent idiosyncratic shocks. It is shown that for all stochastic processes for aggregate income and asset prices, the process for aggregate income can be split into processes for individual incomes such that the processes for asset prices are equilibrium processes. The result is obtained for no-trade equilibria. However discontinuities in fundamentals are necessary for discontinuities in assets prices at no-trade equilibria. Therefore economies for which asset prices jump are not included in the analysis. In Heaton \& Lucas (1996) endowments of consumers are hit by both idiosyncratic and aggregate shocks and consumers face transaction costs. It is found that for large transaction costs consumers are not able to smooth income.

In the present paper we study the existence of jumps in assets prices in general equilibrium economies where jumps are generated by the interaction of real markets and incomplete financial markets. We consider pure-exchange economies with infinite horizon, discrete time, uncertainty with a continuum of possible shocks at every date and incomplete financial markets. The evolution of shocks follows a general Markov process. We show: (1) equilibria exist; (2) if financial markets are effectively complete, so equilibrium allocations are Pareto optimal, then prices depend continuously on shocks, and; (3) if financial markets are incomplete, then there is an open set of economies $\mathscr{U}$ such that for every equilibrium of every economy in $\mathscr{U}$ and almost all histories of shocks, prices at every date depend discontinuously on the shock at that date. Hence jumps in prices can be unavoidable.

To the best of our knowledge, the only other paper on existence of equilibrium for economies with infinite horizon, discrete time, uncertainty with a continuum of possible shocks at every date and incomplete financial markets is Araujo, Monteiro \& Pascoa (1996) (AMP). The major differences in terms of assumptions are: (1) in AMP the probability dis- 
tribution on the set of possible shocks at every date is the Lebesgue measure making the probability distribution independent of the history of shocks, here the probability distribution on the set of possible shocks at date $t$ depends on the shock at date $t-1$; (2) in AMP fundamentals at date $t$ depend on the history of shocks up to and including date $t$, here fundamentals at date $t$ depend on the shock at date $t$; and, (3) in AMP consumption sets are non-negative orthants and utility functions are continuous, here consumption sets are positive orthants and utility functions are twice differentiable functions tending to minus infinity as consumption converges to the boundary of the consumption set. The two first differences (1) and (2) more or less cancel out. However the third difference (3) is important. Indeed a strong form of discounting is needed to get existence of equilibrium in AMP (see Theorem 4.1 in AMP). No such assumption is needed in the present paper because of (3) (see Theorem 1 below).

Continuity of prices is studied in Chichilnisky \& Zhou (1998) and Covarrubias (2010). Pure-exchange economies with one date and uncertainty are considered. The set of possible shocks is a compact subset of some Euclidean space and fundamentals depend continuously on shocks. It is shown that prices depend continuously on shocks in Walrasian equilibria. Optimality of equilibrium allocations for economies with infinite horizon, uncertainty with a finite number of possible shocks and incomplete financial markets is studied in Kubler \& Schmedders (2003). It is shown that if an equilibrium allocation is Pareto optimal, then equilibrium prices and allocations at every date only depend on the shock at that date, and that generically equilibrium allocations are not Pareto optimal. Multiplicity of equilibria is studied in Mas-Colell (1991). Pure-exchange economies with two dates and uncertainty with a continuum of possible shocks at the second date are considered. It is shown that if financial market are incomplete, then some economies have a continuum of equilibria and that in these equilibria prices of goods at the second date depend discontinuously on shocks even though fundamentals depend continuously on shocks.

The present paper is organized as follows: in Section 2 the set-up is introduced and the assumptions are stated; in Section 3 results on existence of equilibrium and results on economies without and with jumps in prices are stated and established; and, Section 4 contains some final remarks.

\section{The model}

Below we introduce our set-up, state the consumer problem and state our assumptions. 


\section{Set-up}

Consider a pure exchange economy with infinite horizon and uncertainty. There is an infinite number of dates $t \in \mathbb{N}_{0}=\{0,1,2, \ldots\}$. The set of possible shocks at every date is $S=[0,1]$ with $s_{t} \in S$ and the shock at the first date is $s_{0} \in S$. The evolution of shocks as time passes is described by a bounded and measurable time independent transition density $\pi: S \times S \rightarrow \mathbb{R}_{++}$ with $\int \pi\left(s_{t+1}, s_{t}\right) d s_{t+1}=1$ for almost all $s_{t}$. Let $s^{t}=\left(s_{t}, \ldots, s_{1}\right)$ denote the history of shocks up to and including date $t$ and let $S^{t}$ denote the set of possible histories of shocks up to and including date $t$.

There are finite numbers of goods $\ell$ with $h \in\{1, \ldots, \ell\}$, consumers $m$ with $i \in\{1, \ldots, m\}$ and short-lived real assets $n$ with $j \in\{1, \ldots, n\}$. Consumers have identical consumption sets $X=\mathbb{R}_{++}^{\ell}$ and discount factors $\left.\rho \in\right] 0,1[$. Consumers are described by their time independent endowments $\omega_{i}: S \rightarrow X$, state utility functions $u_{i}: X \rightarrow \mathbb{R}$ and lower bounds on short sales of assets $\delta_{i} \in \mathbb{R}_{++}^{n}$. Assets are described by their time independent dividends $a_{j}: S \rightarrow \mathbb{R}_{+}^{\ell} \backslash\{0\}$. An economy is a list of consumers and a list of assets $\mathscr{E}=\left(\left(\omega_{i}, u_{i}, \delta_{i}\right),\left(a_{j}\right)\right)$.

Prices of goods and assets are normalized such that they sum to one. Let $\triangle=\{v \in$ $\left.\mathbb{R}_{+}^{\ell+n} \mid \sum_{k} v^{k}=1\right\}$ be the set of normalized prices. A price system is a collection of maps $(p, q)=\left(p_{t}, q_{t}\right)$ where $\left(p_{t}, q_{t}\right): S^{t} \rightarrow \triangle$ for all $t$. A consumption bundle is a collection of maps $x_{i}=\left(x_{i}^{t}\right)$ with $x_{i}^{t}: S^{t} \rightarrow X$ for all $t$. An allocation $x=\left(x_{i}\right)$ is a list of individual consumption bundles. A portfolio is a collection of maps $\theta_{i}=\left(\theta_{i}^{t}\right)$ with $\theta_{i}^{t}: S^{t} \rightarrow-\left\{\delta_{i}\right\}+$ $\mathbb{R}_{+}^{n}$ for all $t$. A consumption plan is a pair of collections of maps $\left(x_{i}, \theta_{i}\right)$.

Financial markets are incomplete for two reasons. First the set of assets is finite and the set of shocks is infinite. Second there are bounds on short sales.

\section{Consumers}

At date $t$ for a history of shocks $s^{t}$ let $\theta_{i}^{t-1}\left(s^{t-1}\right) \in-\left\{\delta_{i}\right\}+\mathbb{R}_{+}^{n}$ be the portfolio from date $t-1$ and $\left(p_{t}\left(s^{t}\right), q_{t}\left(s^{t}\right)\right) \in \triangle$ the prices. Then at date $t$ for a history of shocks $s^{t}$ the budget constraint of consumer $i$ is

$$
p_{t}\left(s^{t}\right) \cdot\left(x_{i}^{t}\left(s^{t}\right)-\omega_{i}\left(s_{t}\right)\right)+\sum_{j} q_{t}^{j}\left(s^{t}\right) \theta_{i}^{t j}\left(s^{t}\right) \leq \sum_{j} p_{t}\left(s^{t}\right) \cdot a_{j}\left(s_{t}\right) \theta_{i}^{t-1 j}\left(s^{t-1}\right) .
$$

Assume $u_{i}$ is continuous, $x_{i}$ is measurable and there exists a compact set $C \subset X$ such that $x_{i}^{t}\left(s^{t}\right) \in C$ for all $t$ and almost all $s^{t}$. Then the expected utility of consumer $i$ is

$$
\begin{aligned}
U_{i}\left(x_{i}\right)=u_{i}\left(x_{i}^{0}\right) & +\rho \int u_{i}\left(x_{i}^{1}\left(s^{1}\right)\right) \pi\left(s_{1}, s_{0}\right) d s^{1} \\
& +\rho^{2} \int u_{i}\left(x_{i}^{2}\left(s^{2}\right)\right) \pi\left(s_{2}, s_{1}\right) \pi\left(s_{1}, s_{0}\right) d s^{2}+\ldots \\
& +\rho^{t} \int u_{i}\left(x_{i}^{t}\left(s^{t}\right)\right) \pi\left(s_{t}, s_{t-1}\right) \ldots \pi\left(s_{1}, s_{0}\right) d s^{t}+\ldots
\end{aligned}
$$


The problem of consumer $i$ is to choose a consumption plan that maximizes expected utility subject to the budget constraints.

\section{Assumptions}

The consumers are assumed to satisfy the following assumptions

(A.1) $\omega_{i} \in C\left(S, \mathbb{R}_{++}^{\ell}\right)$.

(A.2) $u_{i} \in C^{2}(X, \mathbb{R})$ with $D u_{i}\left(x_{i}\right) \in \mathbb{R}_{++}^{\ell}$ and $v^{T} D^{2} u_{i}\left(x_{i}\right) v<0$ for all $x_{i}$ and $v \neq 0$.

(A.3) $\lim _{x_{i k} \rightarrow x_{i}} u_{i}\left(x_{i k}\right)=-\infty$ where $x_{i k} \in X$ for all $k$ and $x_{i} \in \partial X$.

(A.2) states that the utility function is twice differentiable, smoothly strongly monotonic and smoothly strictly concave. (A.3) implies consumption is bounded away from the boundary of the consumption set. The assets are assumed to satisfy the following assumption

(A.4) $a_{j} \in C\left(S, \mathbb{R}_{+}^{\ell} \backslash\{0\}\right)$.

The transition density is assumed to satisfy the following assumption

(A.5) $\pi \in C\left(S \times S, \mathbb{R}_{++}\right)$.

(A.1), (A.4) and (A.5) imply fundamentals depend continuously on shocks.

In order to define a topology on the set of economies satisfying (A1)-(A.5), topologies on endowments, utility functions, assets and transition densities have to be defined. The sets of endowments, assets and transition densities are endowed with the maximum norm topologies. The set of utility functions is endowed with the Whitney $C^{2}$-topology. Finally the set of economies $\mathscr{E}=\left(\left(\omega_{i}, u_{i}, \delta_{i}\right),\left(a_{j}\right)\right)$ is endowed with the product topology.

\section{Equilibria}

Below we state and establish results on existence of equilibrium and jumps in prices.

\section{Existence of equilibrium}

In equilibrium consumers maximize their expected utilities subject to their budget constraints and markets for goods as well as assets clear.

Definition 1 A financial market equilibrium is a price system and a list of individual consumption plans $((\bar{p}, \bar{q}),(\bar{x}, \bar{\theta}))$ such that

- For all $i,\left(\bar{x}_{i}, \bar{\theta}_{i}\right)$ is a solution to the problem of consumer $i$ given $(\bar{p}, \bar{q})$.

- For all $t$ and almost all $s^{t}, \sum_{i} \bar{x}_{i}^{t}\left(s^{t}\right)=\sum_{i} \omega_{i}\left(s_{t}\right)$ and $\sum_{i} \bar{\theta}_{i}^{t}\left(s^{t}\right)=0$. 
Assume portfolio returns are covered by initial endowments for every consumer, all states and all possible portfolios, then there is an equilibrium. The assumption that all possible portfolio returns are covered by initial endowments is very strong. However an example in Mas-Colell \& Zame (1996) shows that the assumption is necessary for existence of financial market equilibrium for economies with non-atomic state spaces.

Theorem 1 For an economy $\mathscr{E}$ suppose that for all $i$ and almost all $s, \omega_{i}(s)-\sum_{j} a_{j}(s) \delta_{i}^{j} \in$ $X$. Then there is a financial market equilibrium $((\bar{p}, \bar{q}),(\bar{x}, \bar{\theta}))$.

Proof: First it has to be shown there exists a compact set $C \subset X$ such that if $((\bar{p}, \bar{q}),(\bar{x}, \bar{\theta}))$ is a financial market equilibrium, then $\bar{x}_{i}^{t}\left(s^{t}\right) \in C$ for all $i$ and $t$ and almost all $s^{t}$. Second it is shown how uncertainty and fundamentals in the present paper can be transformed into uncertainty and fundamentals in AMP. Third the proof of Theorem 4.1 in AMP is applied and it is shown how that an a strong form of discounting used in AMP is not needed because equilibrium consumption is in a compact set $C$.

First: Let $c_{U} \in X$ be defined by $c_{U}^{h}=\max _{s \in S} \sum_{i} \omega_{i}^{h}(s)$ for all $h$. Then $\bar{x}_{i}^{t h}\left(s^{t}\right) \leq c_{U}^{h}$ for all $i, t$ and $h$ and almost all $s^{t}$. Let $\alpha_{i}=\min _{s} u_{i}\left(\omega_{i}(s)-\sum_{h} a_{j}(s) \delta_{i}^{j}\right)$ and $\beta_{i}=\min _{s} u_{i}\left(\omega_{i}(s)\right)$. Then for all $t$ and almost all $s^{t}$ the expected utility from date $t$ and forward is less than or equal to $u_{i}\left(\bar{x}_{i}^{t}\left(s^{t}\right)\right)+\rho u_{i}\left(c_{U}\right)+\rho^{2} u_{i}\left(c_{U}\right)+\ldots$ and larger than or equal to $\alpha_{i}+\rho \beta_{i}+\rho^{2} \beta_{i}+\ldots$. Therefore there exists $c_{L} \in X$ such that $\bar{x}_{i}^{t h}\left(s^{t}\right) \geq c_{L}^{h}$ for all $i, t$ and $h$ and almost all $s^{t}$ because $\limsup \operatorname{sik}_{i k} u_{i} u_{i}\left(x_{i k}\right)=-\infty$ where $x_{i k} \in X$ and $x_{i} \in \partial X$ according to (A.3). Let $C \subset X$ defined by

$$
C=\left\{c \in X \mid \forall h: c^{h} \in\left[c_{L}^{h}, c_{U}^{h}\right]\right\}
$$

then $\bar{x}_{i}^{t}\left(s^{t}\right) \in C$ for all $i$ and $t$ and almost all $s^{t}$.

Second: The economy is transformed into an artificial economy by changing the probability measure on the set of shocks as well as the endowments and the dividends at every date. Suppose the probability measure on the set of shocks at every date is the Lebesgue measure independently of the history of shocks up to that date. Let $\phi: S \times S \rightarrow S$ be a map from shocks at date $t$ and $t-1$ to artificial shocks at date $t$ defined by $\phi\left(s_{t}, s_{t-1}\right)=\operatorname{Prob}\left(s \leq s_{t} \mid s_{t-1}\right)$ so

$$
\phi\left(s_{t}, s_{t-1}\right)=\int_{s \leq s_{t}} \pi\left(s, s_{t-1}\right) d s
$$

Then $\phi\left(\cdot, s_{t-1}\right): S \rightarrow S$ is continuous, strongly monotone and bijective. Let $\psi: S^{2} \rightarrow S$ be defined by $\psi\left(\sigma_{t}, s_{t-1}\right)=s_{t}$ if and only if $\sigma_{t}=\phi\left(s_{t}, s_{t-1}\right)$, so $\psi\left(\cdot, s_{t-1}\right)$ is the inverse of $\phi\left(\cdot, s_{t-1}\right)$. Therefore $\psi\left(\cdot, s_{t-1}\right)$ is continuous, strongly monotone and bijective.

A history of shocks in the original economy $s^{t}$ is transformed into a history of shocks in the artificial economy $\sigma^{t}\left(s^{t}\right)$ as follows: $\sigma_{1}=\phi\left(s_{1}, s_{0}\right), \sigma_{2}=\phi\left(s_{2}, s_{1}\right)$ and so on. Similarly 
a history of shocks of the artificial economy $\sigma^{t}$ is transformed into a history of shocks in the original economy $s^{t}\left(\sigma^{t}\right)$ as follows: $s_{1}=\psi\left(\sigma_{1}, s_{0}\right), s_{2}=\psi\left(\sigma_{2}, s_{1}\right)=\psi\left(\sigma_{2}, \psi\left(\sigma_{1}, s_{0}\right)\right)$ and so on. Let the endowments in the artificial economy $e_{i}^{t}: S^{t} \rightarrow \mathbb{R}_{+}^{\ell}$ at date $t$ be defined by $e_{i}^{0}=\omega_{i}\left(\sigma_{0}\right)$ and $e_{i}^{t}\left(\sigma^{t}\right)=\omega_{i}\left(s_{t}\left(\sigma^{t}\right)\right)$ for all $i$ and $t \geq 1$. Let the dividends in the artificial economy $d_{j}^{t}: S^{t} \rightarrow \mathbb{R}_{+}^{\ell}$ at date $t$ be defined by $d_{j}^{t}\left(\sigma^{t}\right)=a_{j}\left(s_{t}\left(\sigma^{t}\right)\right)$ for all $j$ and $t$.

Third: The proof of Theorem 4.1 in AMP can be applied to the artificial economy with the Lebesgue measure on the set of shocks at every date $t \geq 1, e_{i}=\left(e_{i}^{t}\right)$ as endowments for consumer $i$ and $d_{j}=\left(d_{j}^{t}\right)$ as dividends for asset $j, \mathscr{E} A=\left(\left(e_{i}, u_{i}, \delta_{i}\right),\left(d_{j}\right)\right)$. In the first part of the proof in AMP economies are approximated by economies with finitely many dates and finitely many possible shocks per date. For every $N \in \mathbb{N}$ a truncated artificial economy $\mathscr{E}_{N}^{A}$ with $N+1$ dates and $N$ possible shocks per date is defined. For $S_{N}^{n}=[(n-1) / N, n / N[$ for $n \in\{1, \ldots, N-1\}$ and $S_{N}^{n}=[(n-1) / N, n / N]$ for $n=N$ the set of possible shocks at every date is $\{1, \ldots, N\}$ with $v_{N t} \in\{1, \ldots, N\}$ and $v_{N}^{t} \in\{1, \ldots, N\}^{t}$. For $S_{N}\left(v_{N}^{t}\right)=S_{N}^{n_{1}} \times \ldots \times S_{N}^{n_{t}}$ let endowments $e_{N i}=\left(e_{N i}^{t}\right)$ and dividends $d_{j N}=\left(d_{j N}^{t}\right)$ be defined by

$$
\begin{aligned}
& e_{N i}^{t}\left(v_{N}^{t}\right)=N^{t} \int_{S_{N}\left(v_{N}^{t}\right)} e_{i}^{t}\left(\sigma^{t}\right) d \sigma^{t} \\
& d_{N i}^{t}\left(v_{N}^{t}\right)=N^{t} \int_{S_{N}\left(v_{N}^{t}\right)} d_{j}^{t}\left(\sigma^{t}\right) d \sigma^{t} .
\end{aligned}
$$

For every $N$ the artificial economy with $N+1$ dates and $N$ possible shocks per date has an equilibrium $\left(\left(\bar{p}_{N}, \bar{q}_{N}\right),\left(\bar{x}_{N}, \bar{\theta}_{N}\right)\right)$ according to Radner (1972).

According to Lemma 5.1 in AMP for every $i$ and $N$ there exist Lagrange multipliers $\left(\Lambda_{N i}^{t}\right)$ such that

$$
\begin{aligned}
\left(u_{i}\left(x_{N i}^{0}\right)-\right. & \left.u_{i}\left(\bar{x}_{N i}^{0}\right)\right)+\ldots+\frac{\rho^{N}}{N^{N}} \sum_{v_{N}^{N}}\left(u_{i}\left(x_{N i}^{N}\left(v_{N}^{N}\right)\right)-u_{i}\left(\bar{x}_{N i}^{N}\left(v_{N}^{N}\right)\right)\right) \\
\leq & \Lambda_{N i}^{0} \bar{p}_{N 0} \cdot\left(x_{N i}^{0}-\bar{x}_{N i}^{0}\right)+\ldots+\sum_{v_{N}^{N}} \Lambda_{N i}^{N}\left(v_{N}^{N}\right) \bar{p}_{N N}\left(v_{N}^{N}\right) \cdot\left(x_{N i}^{N}\left(v_{N}^{N}\right)-\bar{x}_{N i}^{N}\left(v_{N}^{N}\right)\right) \\
& +\Lambda_{N i}^{0} \sum_{j} \bar{q}_{N 0}^{j}\left(\theta_{N i}^{0 j}-\bar{\theta}_{N i}^{0 j}\right)+\ldots \\
& +\sum_{v_{N}^{N-1}} \Lambda_{N i}^{N-1}\left(v_{N}^{N-1}\right) \sum_{j} \bar{q}_{N N-1}^{j}\left(v_{N}^{N-1}\right)\left(\theta_{N i}^{N-1}\left(v_{N}^{N-1}\right)-\bar{\theta}_{N i}^{N-1}\left(v_{N}^{N-1}\right)\right) \\
& -\sum_{v_{N}^{1}} \Lambda_{N i}^{1}\left(v_{N}^{1}\right) \sum_{j} \bar{p}_{N 1} \cdot d_{N j}^{1}\left(v_{N}^{1}\right)\left(\theta_{N i}^{1}\left(v_{N}^{1}\right)-\bar{\theta}_{N i}^{1}\left(v_{N}^{1}\right)\right)-\ldots \\
& -\sum_{v_{N}^{N}} \Lambda_{N i}^{N}\left(v_{N}^{N}\right) \sum_{j} \bar{p}_{N N}\left(v_{N}^{N}\right) \cdot d_{j N}^{N}\left(v_{N}^{N}\right)\left(\theta_{N i}^{N-1 j}\left(v_{N}^{N}\right)-\bar{\theta}_{N i}^{N-1 j}\left(v_{N}^{N}\right)\right)
\end{aligned}
$$


According to Lemma 5.2 in AMP the Lagrange multipliers satisfy that for every $i, N$ and $t$

$$
\frac{\rho^{t}}{N^{t}}\left(u_{i}\left(x_{N i}^{t}\left(v_{N}^{t}\right)\right)-u_{i}\left(\bar{x}_{N i}^{t}\left(v_{N}^{t}\right)\right)\right) \leq \Lambda_{N i}^{t}\left(v_{N}^{t}\right) \bar{p}_{N t}\left(v_{N}^{t}\right) \cdot\left(x_{N i}^{t}\left(v_{N}^{t}\right)-\bar{x}_{N i}^{t}\left(v_{N}^{t}\right)\right) .
$$

Therefore the Lagrange multipliers are defined by

$$
\frac{\rho^{t}}{N^{t}} D u_{i}\left(\bar{x}_{N i}^{t}\left(v_{N}^{t}\right)\right)=\Lambda_{N i}^{t}\left(v_{N}^{t}\right) \bar{p}_{N t}\left(v_{N}^{t}\right)
$$

In Lemma 5.3 in AMP a strong form of discounting is used to show that for the map $\lambda_{N i}^{t}$ : $S^{t} \rightarrow \mathbb{R}_{+}$defined by $\lambda_{N i}^{t}\left(s^{t}\right)=N^{t} \Lambda_{N i}^{t}\left(v_{N}^{t}\right)$ for $s^{t} \in S_{N}^{t}\left(v_{N}^{t}\right)$ the sequence of maps $\left(\lambda_{N i}^{t}\right)$ is uniformly bounded in $N$ for all $i$. Below another proof is provided. In the proof it is used that $\bar{x}_{N i}^{t} \in C$ for every $i, N$ and $t$, but the strong form of discounting used in AMP is not used.

For every $t \leq N-1$ consider the following problem for consumer $i$

$$
\begin{aligned}
\max _{\left(z_{i}, \psi_{i}^{j}\right)} & \frac{\rho^{t}}{N^{t}} u_{i}\left(\bar{x}_{N i}^{t}\left(v_{N}^{t}\right)+z_{i}\right)+\frac{\rho^{t+1}}{N^{t+1}} \sum_{v_{N t+1}} u_{i}\left(\bar{x}_{N i}^{t+1}\left(v_{N}^{t+1}\right)+d_{N j}^{t+1} \psi_{i}^{j}\right) \\
\text { s.t. } & \left\{\begin{aligned}
\bar{p}_{N t}\left(v_{N}^{t}\right) \cdot z_{i}+\bar{q}_{N t}^{j}\left(v_{N}^{t}\right) \psi_{i}^{j}=0 \\
\psi_{i}^{j} \geq-\delta_{i}^{j}-\bar{\theta}_{N i}^{t j}\left(v_{N}^{t}\right) .
\end{aligned}\right.
\end{aligned}
$$

The solution is $z_{i}=0$ and $\psi_{i}^{j}=0$ for all $i$ because $\left(\left(\bar{p}_{N}, \bar{q}_{N}\right),\left(\bar{x}_{N}, \bar{\theta}_{N}\right)\right)$ is an equilibrium. For every $j$ there is an $i$ such that $\bar{\theta}_{N i}^{j}\left(v_{N}^{t}\right)>-\delta_{i}^{j}$ because $\sum_{i} \bar{\theta}_{N i}^{j}\left(v_{N}^{t}\right)=0$ and $\delta_{i}^{j}>0$. For every $j$ let $i(j)$ denote an $i$ with $\bar{\theta}_{N i}^{j}\left(v_{N}^{t}\right)>-\delta_{i}^{j}$. The first-order conditions for the problem for consumer $i(j)$ at $z_{i(j)}=0$ and $\psi_{i(j)}^{j}=0$ are

$$
\begin{aligned}
\frac{\rho^{t}}{N^{t}} D u_{i(j)}\left(\bar{x}_{N i(j)}^{t}\left(v_{N}^{t}\right)\right)-\mu_{i(j)} \bar{p}_{N t}\left(v_{N}^{t}\right) & =0 \\
-\mu_{i(j)} \bar{q}_{N t}^{j}\left(v_{N}^{t}\right)+\frac{\rho^{t+1}}{N^{t+1}} \sum_{v_{N t+1}} D u_{i(j)}\left(\bar{x}_{N i(j)}^{t+1}\left(v_{N}^{t+1}\right)\right) \cdot d_{N j}\left(v_{N}^{t}\right) & =0 .
\end{aligned}
$$

Let $\mathbf{1}_{\ell}$ be the vector in $\mathbb{R}^{\ell}$ with every coordinate equal to one. Then

$$
\bar{q}_{N t}^{j}\left(v_{N}^{t}\right)=\frac{\rho}{N} \frac{\sum_{v_{N t+1}} D u_{i(j)}\left(\bar{x}_{N i(j)}^{t+1}\left(v_{N}^{t+1}\right)\right) \cdot d_{N j}\left(v_{N}^{t}\right)}{D u_{i(j)}\left(\bar{x}_{N i(j)}^{t}\left(v_{N}^{t}\right)\right) \cdot \mathbf{1}_{\ell}} \bar{p}_{N t}\left(v_{N}^{t}\right) \cdot \mathbf{1}_{\ell}
$$

Since $\bar{p}_{N t}\left(v_{N}^{t}\right) \cdot \mathbf{1}_{\ell}+\bar{q}_{N t}\left(v_{N}^{t}\right) \cdot \mathbf{1}_{n}=1$,

$$
\bar{p}_{N t}\left(v_{N}^{t}\right) \cdot \mathbf{1}_{\ell}=\frac{1}{1+\sum_{j} \frac{\rho}{N} \frac{\sum_{v_{N t+1}} D u_{i(j)}\left(\bar{x}_{N i(j)}^{t+1}\left(v_{N}^{t+1}\right)\right) \cdot d_{N j}\left(v_{N}^{t}\right)}{D u_{i(j)}\left(\bar{x}_{N i(j)}^{t}\left(v_{N}^{t}\right)\right) \cdot \mathbf{1}_{\ell}}}
$$


SO

$$
\frac{1}{1+\rho \frac{\max _{i, c \in C, j, s} D u_{i}(c) \cdot a_{j}(s)}{\min _{i, c \in C} D u_{i}(c) \cdot \mathbf{1}_{\ell}}} \leq \bar{p}_{N}^{t} \cdot \mathbf{1}_{\ell} \leq \frac{1}{1+\rho \frac{\min _{i, c \in C, j, s} D u_{i}(c) \cdot a_{j}(s)}{\max _{i, c \in C} D u_{i}(c) \cdot \mathbf{1}_{\ell}}}
$$

Since

$$
N^{t} \Lambda_{N i}\left(v_{N}^{t}\right)=\frac{\rho^{t} D u_{i}\left(\bar{x}_{N i}^{t}\left(v_{N}^{t}\right)\right) \cdot \mathbf{1}_{\ell}}{\bar{p}_{N}^{t}\left(v_{N}^{t}\right) \cdot \mathbf{1}_{\ell}}
$$

and

$$
\min _{i, c \in C} D u_{i}(c) \cdot \mathbf{1}_{\ell} \leq D u_{i}\left(\bar{x}_{N i}^{t}\left(v_{N}^{t}\right)\right) \cdot \mathbf{1}_{\ell} \leq \max _{i, c \in C} D u_{i}(c) \cdot \mathbf{1}_{\ell}
$$

the sequence $\left(\lambda_{N i}^{t}\right)$ is uniformly bounded in $N$ for every $i$.

The rest of the proof follows the last part of the proof of Theorem 4.1 in AMP. An equilibrium for the artificial economy is obtained as a limit of the sequence of equilibria for the sequence of truncated artificial economies. Uniform boundedness of the sequences $\left(\lambda_{N i}^{t}\right)$ is used to show that the limit of the sequences of solutions to the problems of the consumers in the truncated artificial economies are solutions to the problems of the consumers in the artificial economy.

Remark: Theorem 4.1 in AMP on existence of equilibrium rests on the assumption that there is $\varepsilon>0$ such that

$$
\min _{s}\left(\omega_{i}^{h}(s)-\sum_{j} a_{j}^{h}(s) \delta_{i}^{j}\right)>\varepsilon
$$

for all $i$ and $h$ and

$$
\rho\left(\varepsilon+\sum_{i} \delta_{i}^{j}\right) \max _{s} a_{j}^{h}(s)<\varepsilon
$$

for all $j$ and $h$. The first part is equivalent to the assumption in Theorem 1 while the second part puts an upper bound on the discount factor. In the present paper the second part of the assumption is not used because of (A.2) and (A.3).

\section{Continuous financial market equilibria}

An allocation is Pareto optimal provided there is no other allocation for which no consumer is worse off and at least one consumer is better off.

Definition 2 A Pareto optimal allocation is an allocation $\tilde{x}$ for which there is no other allocation $x$ with

- $\sum_{i} x_{i}^{t}\left(s^{t}\right) \leq \sum_{i} \omega_{i}\left(s_{t}\right)$ for all $t$ and almost all $s^{t}$.

- $U_{i}\left(x_{i}\right) \geq U_{i}\left(\tilde{x}_{i}\right)$ for all $i$ with " $>$ " for at least one $i$.

In order for jumps to matter for consumers, prices and consumption plans have to be measurable discontinuous. 
Definition 3 A bounded and measurable map $f: S \rightarrow \mathbb{R}$ is (measurable) continuous provided there is a continuous map $g \in C(S, \mathbb{R})$ such that

$$
\int|f(s)-g(s)| d s=0 .
$$

Otherwise it is (measurable) discontinuous.

Remark: A map is measurable continuous provided it can be made continuous by changing it on a set of measure zero. Indeed the map $f: S \rightarrow[0,1]$ defined by

$$
f(s)= \begin{cases}0 & \text { for } s \text { rational } \\ 1 & \text { otherwise }\end{cases}
$$

is measurable continuous, because the set of rationals has measure zero, so $\int|f(s)-1| d s=$ 0 . Therefore random samples from continuous and measurable continuous maps $\left(s_{a}, f\left(s_{a}\right)\right)$ and $\left(s_{a}, g\left(s_{a}\right)\right)$ cannot be distinguished.

Remark: A map is measurable discontinuous provided it has a jump. Indeed the map $f$ : $S \rightarrow[0,1]$ defined by

$$
h(s)= \begin{cases}0 & \text { for } s \in\left[0, \frac{1}{2}\right] \\ 1 & \text { for } \left.s \in] \frac{1}{2}, 1\right]\end{cases}
$$

is measurable discontinuous. For the sequence of maps $\left(g_{n}\right)$ with $g_{n}: S \rightarrow[0,1]$ defined by

$$
g_{n}(s)=\left\{\begin{array}{cl}
0 & \text { for } s \in\left[0, \frac{1}{2}-\frac{1}{n+1}[\right. \\
\frac{n+1}{2} s-\frac{n-1}{4} & \text { for } s \in\left[\frac{1}{2}-\frac{1}{n+1}, \frac{1}{2}+\frac{1}{n+1}\right] \\
1 & \text { for } \left.s \in] \frac{1}{2}+\frac{1}{n+1}, 1\right]
\end{array}\right.
$$

$\lim _{n \rightarrow \infty} \int\left|h(s)-g_{n}(s)\right|=0$ because $\int\left|h(s)-g_{n}(s)\right| d s=1 /(2(n+1))$. Therefore random samples from measurable discontinuous maps $\left(s_{a}, h\left(s_{a}\right)\right)$ and continuous maps $\left(s_{a}, g_{n}\left(s_{a}\right)\right)$ can be very hard to distinguish. However from a mathematical point of view it is easier to study measurable discontinuous maps than continuous maps with very steep slopes.

For incomplete financial markets there is no reason to expect equilibrium prices and consumption plans to depend continuously on shocks. However equilibrium prices and consumption bundles are time independent and depend continuously on shocks provided the equilibrium allocation is Pareto optimal. Equilibrium portfolios need not depend continuously on shocks in case dividends of assets are collinear. 
Theorem 2 For an economy $\mathscr{E}$ let $((\bar{p}, \bar{q}),(\bar{x}, \bar{\theta}))$ be a financial market equilibrium. Suppose $\bar{x}$ is Pareto optimal. Then $\left(\bar{p}_{t}, \bar{q}_{t}\right): S^{t} \rightarrow \triangle$ and $\bar{x}_{t}: S^{t} \rightarrow X^{m}$ are continuous for all $t$. Indeed there are continuous functions $(p, q): S \rightarrow \triangle$ and $x: S \rightarrow X^{m}$ such that

$$
\begin{aligned}
\left(\bar{p}^{t}\left(s^{t}\right), \bar{q}\left(s^{t}\right)\right) & =\left(p\left(s_{t}\right), q\left(s_{t}\right)\right) \\
\bar{x}^{t}\left(s^{t}\right) & =x\left(s_{t}\right)
\end{aligned}
$$

for all $t$ and almost all $s^{t}$.

Proof: For every $r \in \mathbb{R}_{++}^{\ell}, \bar{c} \in X^{m}$ with $\sum_{i} c_{i} \leq r$ and $\lambda \in \mathbb{R}_{++}^{m}$ the set

$$
\left\{c \in X^{m} \mid \sum_{i} c_{i} \leq r \text { and } \sum_{i} \lambda_{i} u_{i}\left(c_{i}\right) \geq \sum_{i} \lambda_{i} u_{i}\left(\bar{c}_{i}\right)\right\}
$$

is compact because for all $i, u_{i}$ is continuous according to (A.2) and $\lim _{x_{i k} \rightarrow x_{i}} u_{i}\left(x_{i k}\right)=-\infty$ according to (A.3). Therefore for every $s \in S$ and $\lambda \in \mathbb{R}_{++}^{m}$ there exists a unique solution to the following problem

$$
\begin{array}{ll}
\max _{c} & \sum_{i} \lambda_{i} u_{i}\left(c_{i}\right) \\
\text { s.t. } & \sum_{i} c_{i} \leq \sum_{i} \omega_{i}(s)
\end{array}
$$

because $u_{i}$ is continuous and strictly concave according to (A.2). Let $c: S \times \mathbb{R}_{++}^{m} \rightarrow X^{m}$ be the solution to the problem. Then $c: S \times \mathbb{R}_{++}^{m} \rightarrow X^{m}$ is continuous according to Berge's maximum theorem.

For every $\lambda \in \mathbb{R}_{++}^{m}$ consider the planner problem

$$
\begin{aligned}
\max _{x^{t}} & \sum_{i} \lambda_{i} U_{i}\left(x_{i}^{t}\left(s^{t}\right)\right) \\
\text { s.t. } & \sum_{i} x_{i}^{t}\left(s^{t}\right) \leq \sum_{i} \omega_{i}\left(s_{t}\right) \text { for all } t \text { and almost } s^{t}
\end{aligned}
$$

Then the allocation $c(\lambda)$ is a solution to the planner problem if and only if $c^{t}\left(s^{t}, \lambda\right)=c\left(s_{t}, \lambda\right)$ for all $t$ and almost all $s^{t}$. Next it is shown that $x$ with $U_{i}\left(x_{i}\right)=-\infty$ for some $i$ is neither a solution to the planner problem nor Pareto optimal.

Let $\omega_{L} \in X$ be defined by $\omega_{L}^{h}=(1 / m) \min _{s \in S} \sum_{i} \omega_{i}^{h}(s)$. For $x$ if $U_{i}(x)=-\infty$ for some $i$, then $x$ is not a solution to the planner problem for any $\lambda \in \mathbb{R}_{++}^{m}$ because $u_{i}\left(\omega_{L}\right)+\rho u_{i}\left(\omega_{L}\right)+$ $\rho^{2} u_{i}\left(\omega_{L}\right)+\ldots>-\infty$ for all $i$. For $x$ if $U_{i}\left(x_{i}\right)=-\infty$ for all $i$, then $x$ is not Pareto optimal because $u_{i}\left(\omega_{L}\right)+\rho u_{i}\left(\omega_{L}\right)+\rho^{2} u_{i}\left(\omega_{L}\right)+\ldots>-\infty$ for all $i$. For $x$ if $U_{i}\left(x_{i}\right)=-\infty$ for some $i$ and $U_{i^{\prime}}\left(x_{i^{\prime}}\right)>-\infty$ for some $i^{\prime}$, then $x$ is not Pareto optimal because $x^{\prime}$ defined by $x_{i}^{\prime}=(1 / m) x_{i}$ and $x_{i^{\prime}}^{\prime}=x_{i^{\prime}}+(1 / m) x_{i}$ for all $i^{\prime} \neq i$ makes all consumers $i^{\prime}$ with $U_{i^{\prime}}\left(x_{i^{\prime}}\right)>\infty$ better off and no 
consumer worse off. Next it is shown that $x$ is Pareto optimal if and only if $x_{i}^{t}\left(s^{t}\right)=c_{i}\left(s_{t}, \bar{\lambda}\right)$ for all $t$ and $i$ and almost all $s^{t}$.

Suppose $x$ is not Pareto optimal. Then there exists another allocation $x^{\prime}$ such that $U_{i}\left(x_{i}^{\prime}\right) \geq U_{i}\left(x_{i}\right)$ for all $i$ with at least one strict inequality. Therefore $x$ is not a solution the planner problem for any $\lambda \in \mathbb{R}_{++}^{m}$. Hence if $x$ is a solution to the planner problem, then $x$ is Pareto optimal. Suppose $x$ is not a solution to the planner problem for any $\lambda \in \mathbb{R}_{++}^{m}$. Then $\sum_{i} \lambda_{i} U_{i}\left(c_{i}(\lambda)\right)>\sum_{i} \lambda_{i} U_{i}\left(x_{i}\right)$ for all $\lambda \in \mathbb{R}_{++}^{m}$. Let $\mu: \mathbb{R}_{++}^{m} \rightarrow \mathbb{R}_{++}$be defined by

$$
\mu(\lambda)=\frac{1}{m} \sum_{i} \lambda_{i}\left(U_{i}\left(c_{i}(\lambda)\right)-U_{i}\left(x_{i}\right)\right)
$$

Then $\mu: \mathbb{R}_{++}^{m} \rightarrow \mathbb{R}_{++}$is continuous. For $\mathscr{S}_{++}^{m-1}=\left\{\lambda \in \mathbb{R}_{++}^{m} \mid\|\lambda\|=1\right\}$ let $\Gamma: \mathscr{S}_{++}^{m-1} \rightarrow \mathbb{R}^{m}$ be defined by $\Gamma_{i}(\lambda)=U_{i}\left(c_{i}(\lambda)\right)-U_{i}\left(x_{i}\right)-\mu(\lambda)$, then: $\Gamma$ is continuous; there exists $\alpha \in \mathbb{R}$ such that $\Gamma_{i}(\lambda) \leq \alpha$ for all $i$ and $\lambda ; \lambda \cdot \Gamma(\lambda)=0$ for all $\lambda$; and, $\lim _{\lambda_{k} \rightarrow \lambda}\left\|\Gamma\left(\lambda_{k}\right)\right\|=\infty$ for all $\lambda \in \partial \mathscr{S}_{++}^{m-1}$. Therefore there exists $\bar{\lambda} \in \mathscr{S}_{++}^{m-1}$ such that $\Gamma(\bar{\lambda})=0$ so $U_{i}\left(c_{i}(\bar{\lambda})\right)>U_{i}\left(x_{i}\right)$ for all $i$ so $x$ is not Pareto optimal. Hence if $x$ is Pareto optimal, then $x$ is a solution to the planner problem for some $\lambda \in \mathscr{S}_{++}^{m}$. All in all, $x$ is Pareto optimal if and only if $x_{i}^{t}\left(s^{t}\right)=c_{i}\left(s_{t}, \bar{\lambda}\right)$ for all $t$ and $i$ and almost all $s^{t}$ so there exists $\lambda \in \mathscr{S}_{++}^{m-1}$ such that $\bar{x}^{t}\left(s^{t}\right)=c\left(s_{t}, \lambda\right)$ for all $t$ and almost all $s^{t}$.

Let $f: S \rightarrow \mathbb{R}_{++}^{\ell}$ and $g: S \rightarrow \mathbb{R}_{++}^{n}$ be defined by

$$
\begin{aligned}
f(s) & =D u_{i}\left(c_{i}(s, \lambda)\right) \\
g^{j}(s) & =\rho \int D u_{i}\left(c_{i}\left(s^{\prime}, \lambda\right)\right) \cdot a_{j}\left(s^{\prime}\right) \pi\left(s^{\prime}, s\right) d s^{\prime} .
\end{aligned}
$$

Then $(\bar{p}, \bar{q})$ satisfies

$$
\begin{aligned}
& \bar{p}_{t}\left(s^{t}\right)=\frac{1}{\sum_{h} f^{h}\left(s_{t}\right)+\sum_{j} g^{j}\left(s_{t}\right)} f\left(s_{t}\right) \\
& \bar{q}_{t}\left(s^{t}\right)=\frac{1}{\sum_{h} f^{h}\left(s_{t}\right)+\sum_{j} g^{j}\left(s_{t}\right)} g\left(s_{t}\right)
\end{aligned}
$$

for all $t$ and almost all $s^{t}$. Therefore $\bar{p}$ and $\bar{q}$ are continuous.

Q.E.D.

Remark: In Elul (1999) financial markets are denoted effectively complete provided equilibrium allocations are Pareto optimal. According to Theorem 2 if financial markets are effectively complete, then consumers are able to smooth consumption across shocks such that jumps are eliminated.

\section{Recurrent discontinuous financial market equilibria}

For an equilibrium, there are recurrent jumps provided that for all dates and histories of shocks $s^{t-1}$, prices and consumption bundles at date $t$ depend discontinuously on the shock 
$s_{t}$.

Definition $4 A$ recurrent discontinuous financial market equilibrium is a financial market equilibrium $((\bar{p}, \bar{q}),(\bar{x}, \bar{\theta}))$ such that for all $t$ and almost all $s^{t-1}$,

$$
\left(\bar{p}_{t}, \bar{q}_{t}, \bar{x}^{t}\right)\left(\cdot, s^{t-1}\right): S \rightarrow \triangle \times X^{m}
$$

is discontinuous.

Suppose a financial market equilibrium is recurrent discontinuous. Then for a fixed history of shocks $s^{t-1}$ the relation between the shock $s_{t}$ at date $t$ and prices and consumption bundles at date $t$ can be as illustrated below in Figure 1.

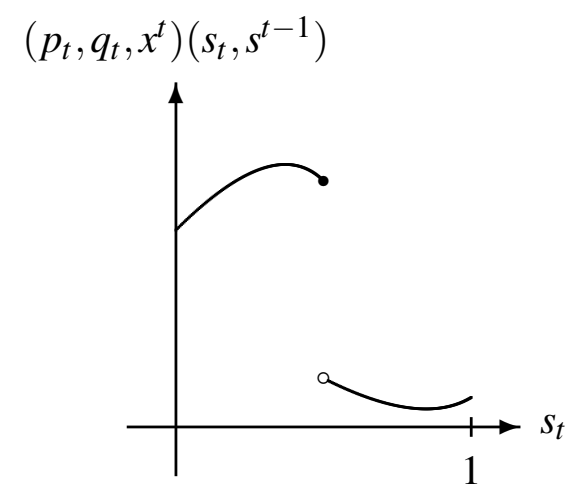

Figure 1: jumps in prices and consumption bundles

There is an open set of economies such that for all equilibria of all economies in that set, all endogenous variables are recurrent discontinuous even though fundamentals depend continuously on shocks.

Theorem 3 There is an open set of economies $\mathscr{U}$, such that every financial market equilibrium $((\bar{p}, \bar{q}),(\bar{x}, \bar{\theta}))$ for every economy $\mathscr{E}$ in $\mathscr{U}$ is recurrently discontinuous.

Proof: Suppose $\left(e_{i}, u_{i}\right)$, where $e_{i} \in X$ for all $i$, is a pure-exchange economy with $\ell$ goods and $m$ consumers and at least two regular Walrasian equilibria. See Ghiglino \& Tvede (1997) for details on how to construct such an economy. Let $c$ and $c^{\prime}$ be two different equilibrium allocations associated with two different regular equilibria of $\left(e_{i}, u_{i}\right)$.

For all $i$ let $\omega_{i}: S \rightarrow X$ be defined by

$$
\omega_{i}(s)=\left\{\begin{array}{cc}
(1-2 s) c_{i}+(2 s) e_{i} & \text { for } s \in\left[0, \frac{1}{2}\right] \\
(2-2 s) e_{i}+(2 s-1) c_{i}^{\prime} & \text { for } \left.s \in] \frac{1}{2}, 1\right] .
\end{array}\right.
$$


Then the pure-exchange economy $\left(\omega_{i}(s), u_{i}\right)$ has a unique regular Walrasian equilibrium for $s \in\{0,1\}$, because endowments are Pareto optimal for $s \in\{0,1\}$, and at least two regular Walrasian equilibria for $s=1 / 2$. Let $W:[0,1] \rightarrow \triangle$ be the Walras correspondence. Then there is no continuous selection from the Walras correspondence. Moreover there exists a neighborhood $\mathscr{N}$ of $\left(\omega_{i}, u_{i}\right)$ such that if $\left(\omega_{i}^{\prime}, u_{i}^{\prime}\right) \in \mathscr{N}$, then there is no continuous selection from the Walras correspondence for $\left(\omega_{i}^{\prime}, u_{i}^{\prime}\right)$.

Suppose the discount factor $\rho$, the number of assets $n$, the dividends of the assets $\left(a_{j}\right)$ and the lower bounds on short sales $\left(\delta_{i}\right)$ are arbitrary and fixed such that $\min _{s} \omega_{i}^{h}(s)-$ $\sum_{j} a_{j}^{h}(s) \delta_{i}^{j}>0$ for all $i$ and $h$. Consider a family of economies $\mathscr{E} \tau=\left(X, \rho,\left(\omega_{i}, u_{i}\right),\left(a_{j}^{\tau}\right)\right)$ parametrized by $\tau \in[0,1]$ and defined by $a_{j}^{\tau}=\tau a_{j}$ for all $\tau \in[0,1]$. According to Theorem 1 the economy $\mathscr{E}^{\tau}$ has a financial market equilibrium for every $\tau$.

For every pair of consumers $i$ and $i^{\prime}$, date $t$, asset $j$ and $\tau$ and almost all histories of shocks $s^{t}$ the vectors $D u_{i}\left(\bar{x}_{i}^{\tau t}\left(s^{t}\right)\right)$ and $D u_{i^{\prime}}\left(\bar{x}_{i^{\prime}}^{\tau t}\left(s^{t}\right)\right)$ are collinear. For an arbitrary $i$, all $t$ and $\tau$ and almost all $s^{t}$ let $f_{t}^{\tau}: S^{t} \rightarrow \mathbb{R}_{++}^{\ell}$ be defined by

$$
f_{t}^{\tau}\left(s^{t}\right)=D u_{i}\left(\bar{x}_{i}^{\tau t}\left(s^{t}\right)\right)
$$

For all $t, j$ and $\tau$ and almost all $s^{t}$, there exists an $i$ such that $\bar{\theta}_{i}^{\tau j}\left(s^{t}\right)>-\delta_{i}^{j}$ because $\delta_{i}^{j}>0$ for all $i$ and $\sum_{i} \bar{\theta}_{i}^{\tau j}\left(s^{t}\right)=0$ for all $t$ and almost all $s^{t}$. For all $j, t$ and $\tau$, any $i$ with $\bar{\theta}_{i}^{\tau t j}\left(s^{t}\right)>$ $-\delta_{i}^{j}$ and almost all $s^{t}$ let $g_{t}^{\tau j}: S^{t} \rightarrow \mathbb{R}_{++}^{n}$ be defined by

$$
g_{t}^{\tau j}\left(s^{t}\right)=\rho \int D u_{i}\left(\bar{x}_{i}^{\tau t+1}\left(s^{t+1}\right)\right) \cdot a_{j}^{\tau}\left(s^{t+1}\right) \pi\left(s_{t+1}, s_{t}\right) d s_{t+1} .
$$

Then for all $t$ and almost all $s^{t}$

$$
\begin{aligned}
\bar{p}_{t}^{\tau}\left(s^{t}\right) & =\frac{1}{\sum_{h} f_{t}^{\tau j}\left(s^{t}\right)+\sum_{j} g_{t}^{\tau k}\left(s^{t}\right)} f_{t}^{\tau}\left(s^{t}\right) \\
\bar{q}_{t}^{\tau j}\left(s^{t}\right) & =\frac{1}{\sum_{h} f_{t}^{\tau j}\left(s^{t}\right)+\sum_{j} g_{t}^{\tau j}\left(s^{t}\right)} g_{t}^{\tau j}\left(s^{t}\right)
\end{aligned}
$$

for all $j$.

According to the proof of Theorem 1 there exists $c_{L}, c_{U} \in X$ such that if $\left(\left(\bar{p}^{\tau}, \bar{q}^{\tau}\right),\left(\bar{x}^{\tau}, \bar{\theta}^{\tau}\right)\right)$ is a financial market equilibrium for $\mathscr{E}^{\tau}$, then $\bar{x}_{i}^{\tau t}\left(s^{t}\right) \in C$ for all $i, t$ and $\tau \in[0, \bar{\tau}]$ and almost all $s^{t}$. Therefore asset prices converge uniformly to zero as $\tau$ converges to zero

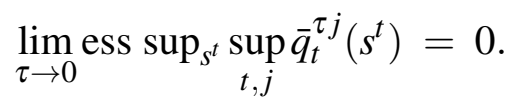

Moreover $\bar{\theta}_{i}^{\tau t j}\left(s^{t}\right) \in\left[-\delta_{i}^{j}, 1+\sum_{i^{\prime} \neq i} \delta_{i^{\prime}}^{j}\right]$ for all $i, t$ and $j$ and almost all $s^{t}$ because $\bar{\theta}_{i}^{\tau t j}\left(s^{t}\right) \geq$ $-\delta_{i}^{j}$ for all $i, t$ and $j$ and almost all $s^{t}$ and $\sum_{i} \bar{\theta}_{i}^{\tau t j}\left(s^{t}\right)=0$ for all $t$ and $j$ and almost all $s^{t}$. 
Hence the real dividends and prices of portfolios converge uniformly to zero as $\tau$ converges to zero

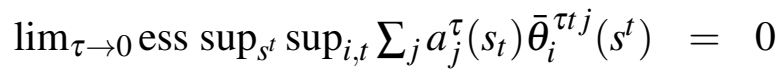

$$
\begin{aligned}
& \lim _{\tau \rightarrow 0} \operatorname{ess} \sup _{s^{t}} \sup _{i, t} \sum_{j} \bar{q}_{t}^{\tau j}\left(s^{t}\right) \bar{\theta}_{i}^{\tau t j}\left(s^{t}\right)=0 .
\end{aligned}
$$

For all $t$ and almost all $s^{t}$ consider a family of pure-exchange economies with income transfers parametrized by $s_{t}$

$$
\mathscr{E}_{t s^{t-1}}^{\tau}\left(s_{t}\right)=\left(\omega_{i}\left(s_{t}\right)+\sum_{j} a_{j}^{\tau}\left(s_{t}\right) \bar{\theta}_{i}^{\tau t-1 j}\left(s^{t-1}\right), u_{i},-\sum_{j} \bar{q}_{t}^{\tau j}\left(s^{t}\right) \bar{\theta}_{i}^{\tau t j}\left(s^{t}\right)\right),
$$

where $\omega_{i}\left(s_{t}\right)+\sum_{j} a_{j}^{\tau}\left(s_{t}\right) \bar{\theta}_{i}^{\tau t-1 j}\left(s^{t-1}\right)$ is the endowment of consumer $i$ and $-\sum_{j} \bar{q}_{t}^{\tau j}\left(s^{t}\right) \bar{\theta}_{i}^{\tau t j}\left(s^{t}\right)$ is the income transfer received by consumer $i$. The equilibrium correspondence $W_{t s^{t-1}}^{\tau}: S \rightarrow$ $\mathbb{R}_{+}^{\ell}$ is defined by $v \in W_{t s^{t-1}}^{\tau}\left(s_{t}\right)$ if and only if $\sum_{h} v_{h}=1-\sum_{j} \bar{q}_{t}^{\tau j}\left(s^{t}\right)$ and there exists $\left(c_{i}\right)$ such that $c_{i}$ is a solution to

$$
\begin{array}{ll}
\max _{c} & u_{i}(c) \\
\text { s.t. } & v \cdot c \leq v \cdot\left(\omega_{i}\left(s_{t}\right)+\sum_{j} a_{j}^{\tau}\left(s_{t}\right) \bar{\theta}_{i}^{\tau t-1 j}\left(s^{t-1}\right)\right)-\sum_{j} \bar{q}_{t}^{\tau j}\left(s^{t}\right) \bar{\theta}_{i}^{\tau t j}\left(s^{t}\right)
\end{array}
$$

for all $i$ and $\sum_{i} c_{i}=\sum_{i} \omega_{i}\left(s_{t}\right)$. Therefore $\bar{p}_{t}^{\tau}\left(s^{t}\right) \in W_{t s^{t-1}}^{\tau}\left(s_{t}\right)$ for all $t$ and $\tau$ and almost all $s^{t}$. There exists $\bar{\tau} \in] 0,1]$ such that for all $\tau \in] 0, \bar{\tau}$ [ there is no continuous selection from $E_{t s^{t-1}}^{\tau}$ for all $t$ and almost all $s^{t-1}$ because there is no continuous selection from $W$. Q.E.D.
.

Remark: In the proof of Theorem 3: first pure-exchange economies without financial markets are constructed such that all endogenous variables are recurrent discontinuous; and, second financial markets, where assets have small dividends, are added so the lower bounds on short sales ensures that all endogenous variables are recurrent discontinuous. In the appendix we present an example of a pure-exchange economy with financial markets and finite time horizon where all endogenous variables are discontinuous and lower bounds on short sales are not binding.

Theorem 3 implies that if financial markets are incomplete, then jumps in both prices and consumption bundles can be unavoidable. In the proof of Theorem 3, it is important how endowments depend on shocks, but not how dividends depend on shocks. Therefore we argue that jumps are generated by the interaction of real and financial markets.

\section{Final remarks}

In the present paper we have provided a possible explanation of jumps in asset prices. Whether there are jumps or not depends on whether markets are incomplete or not. Indeed, 
for effectively complete financial markets there are no jumps and for incomplete financial markets jumps can be unavoidable.

The proofs of Theorems 1-3 reveal that it is straightforward to extend the analysis to include long-lived assets and shock dependent state utility functions. It would be nice to extend the analysis to economies with continuous time. However, such an extension is not straightforward because even existence of equilibrium for economies with incomplete financial markets and continuous time is a territory about which little is known.

\section{Appendix: economies with finite time horizon}

\section{Set-up}

Consider a pure exchange economy with finite horizon and uncertainty. There is a finite number of dates $t \in\{0, \ldots, T\}$. The set of possible shocks at every date is $S=[0,1]$ with $s_{t} \in S$ and the shock at the first date is $s_{0} \in S$. The evolution of shocks as time passes is described by a bounded and measurable time independent transition density $\pi: S \times S \rightarrow \mathbb{R}_{++}$.

There are finite numbers of goods $\ell$ with $h \in\{1, \ldots, \ell\}$, consumers $m$ with $i \in\{1, \ldots, m\}$ and real assets $n$ with $j \in\{1, \ldots, n\}$. Consumers have identical consumption sets $X=$ $\left(\mathbb{R}_{++}^{\ell}\right)^{T+1}$. Consumers are described by their endowments $\omega_{i}=\left(\omega_{i}^{t}\right)$ with $\omega_{i}^{t}: S^{t} \rightarrow \mathbb{R}_{++}^{\ell}$, state utility functions $u_{i}: X \rightarrow \mathbb{R}$ and lower bounds on short sales of assets $\delta_{i} \in \mathbb{R}_{++}^{n}$. Assets are described by their dividends $a_{j}=\left(a_{j}^{t}\right)$ with $a_{j}^{t}: S^{t} \rightarrow \mathbb{R}_{+}^{\ell}$ for all $t$. An economy is a list of consumers and a list of assets $\mathscr{E}^{\text {Finite }}=\left(\left(\omega_{i}, u_{i}, \delta_{i}\right),\left(a_{j}\right)\right)$.

\section{Discontinuous financial market equilibria}

Theorem 4 There exists an economy such that if $((\bar{p}, \bar{q}),(\bar{x}, \bar{\theta}))$ is a financial market equilibrium, then $\bar{q}$ is discontinuous in $s^{T}$.

Proof: Consider an economy with three dates $T=2$, one good in each date $\ell=1$, two consumers $m=2$ and one asset $n=1$. The dividend of the asset is supposed to be one unit of the good at the last date and otherwise before the last date. Endowments and asset dividends are assumed to depend on the shock $s$ at the second date $t=1$ and to be independent of the shock at the third date $t=2$. The probability distribution on the set of shocks at date $t=1$ is the Lebesgue measure $\pi(s)=1$ for all $s$.

Endowments at date $t=0$ are supposed to be identical $\omega_{2}^{0}=\omega_{1}^{0}$ and endowments at the 
last two dates are supposed to be reverse in the sense that

$$
\begin{aligned}
& \omega_{2}^{1}(s)=\omega_{1}^{2}(1-s) \\
& \omega_{2}^{2}(s)=\omega_{1}^{1}(1-s) .
\end{aligned}
$$

Similarly, utility functions are supposed to be identical for the first date and reverse for the last two dates such that

$$
u_{2}\left(x^{0}, \alpha, \beta\right)=u_{1}\left(x^{0}, \beta, \alpha\right)
$$

for all positive real numbers $x^{0}, \alpha, \beta>0$. Denote by $\mathscr{E}\left(s ;\left(c_{i}^{0}\right)\right)=\left(e_{i}(s), v_{i}\left(\cdot ; c_{i}^{0}\right)\right)$ the subeconomy with consumers $i \in\{1,2\}$, utility functions $v_{i}\left(\cdot ; c_{i}^{0}\right): \mathbb{R}_{++}^{2} \rightarrow \mathbb{R}$ defined by $v_{i}\left(x_{i}^{1}, x_{i}^{2} ; c_{i}^{0}\right) \equiv$ $u_{i}\left(c_{i}^{0}, x_{i}^{1}, x_{i}^{2}\right)$ and endowments $e_{i}(s) \in \mathbb{R}^{2}$.

For $c_{i}^{0}$ let $f_{i}\left(\cdot ; c_{i}^{0}\right)=\left(f_{i}^{1}\left(\cdot ; c_{i}^{0}\right), f_{i}^{2}\left(\cdot ; c_{i}^{0}\right)\right): \mathbb{R}_{++}^{2} \times \mathbb{R}_{++} \rightarrow \mathbb{R}_{++}^{2}$ denote the demand function for the consumer $i$ of the sub-economy $\mathscr{E}\left(s ;\left(c_{i}^{0}\right)\right)$, so $f_{i}\left(p, p \cdot e_{i}(s) ; c_{i}^{0}\right)$ solves the problem

$$
\begin{aligned}
\max _{\left(x^{1}, x^{2}\right)} & v_{i}\left(x^{1}, x^{2} ; c_{i}\right) \\
\text { s.t. } & p \cdot\left(x-e_{i}(s)\right) \leq 0 .
\end{aligned}
$$

Then $(s, p) \in S \times \mathbb{R}_{++}^{2}$ is an equilibrium for the sub-economy $\mathscr{E}\left(s ;\left(c_{i}^{0}\right)_{i}\right)$ if and only if

$$
f_{1}\left(p, p \cdot e_{1}(s) ; c_{1}^{0}\right)+f_{2}\left(p, p \cdot e_{2}(s) ; c_{2}^{0}\right)=e_{1}(s)+e_{2}(s) .
$$

Clearly $\left(s, p_{1}, p_{2}\right)$ is an equilibrium for $\mathscr{E}\left(s ;\left(c_{i}^{0}\right)\right)$ if and only if $\left(1-s, p_{2}, p_{1}\right)$ is an equilibrium for $\mathscr{E}\left(1-s ;\left(d_{i}^{0}\right)\right)$, where $d_{1}^{0}=c_{2}^{0}$ and $d_{2}^{0}=c_{1}^{0}$.

For equilibrium prices normalized such that the sum equals one let $W: S \rightarrow S \times \mathbb{R}_{++}^{2}$ be the Walras correspondence for the economies $\left(\mathscr{E}\left(s ;\left(c_{i}^{0}\right)\right)\right)$ with $c_{i}^{0}=\omega_{i}^{0}$ for both $i$, so

$$
W(s)=\left\{\left(s, p_{1}, p_{2}\right) \mid\left(s, p_{1}, p_{2}\right) \text { is an equilibrium for } \mathscr{E}\left(s ;\left(\omega_{i}^{0}\right)\right)\right\} .
$$

Suppose that the graph of $W$ is $S$-shaped as shown in Figure 2 and let $r: S \rightarrow \mathbb{R}_{++}^{2}$ be a selection from $W$ such that $r_{1}(s)$ is the lowest equilibrium price for $s<1 / 2, r_{1}(s)=$ $(1 / 2,1 / 2)$ for $s=1 / 2$ and $r_{1}(s)$ is the highest equilibrium price for $s>1 / 2$. In order to construct a financial market equilibrium: let the allocation $\bar{x}$ be defined by $\bar{x}_{i}^{0}=\omega_{i}^{0}$, and $\bar{x}_{i}^{t}(s)=f_{i}^{t}\left(r(s), r(s) \cdot e_{i}(s) ; \omega_{i}^{0}\right)$ for both $i$ and $t \in\{1,2\}$; let the portfolio $\bar{\theta}$ be defined by $\bar{\theta}_{i}^{0}=0$ and

$$
\bar{\theta}_{i}^{1}(s)=\frac{r_{1}(s)}{r_{2}(s)}\left(\omega_{i}^{1}(s)-f_{i}^{1}\left(r(s), r(s) \cdot e_{i}(s) ; \omega_{i}^{0}\right)\right)=f_{i}^{2}\left(r(s), r(s) \cdot e_{i}(s) ; \omega_{i}^{0}\right)-\omega_{i}^{2}(s) ;
$$

let the price system $(\bar{p}, \bar{q})$ be defined by $\bar{p}_{0}=\bar{p}_{1}(s)=\bar{p}_{2}(s)=1$ for all $s, \bar{q}_{1}(s)=r_{2}(s) / r_{1}(s)$ for all $s$ and $\bar{q}_{0}>0$ such that

$$
\int\left(-\bar{q}_{0} \frac{\partial u_{i}\left(\bar{x}_{i}(s)\right)}{\partial x_{i}^{0}}+\bar{q}_{1}(s) \frac{\partial u_{i}\left(\bar{x}_{i}(s)\right)}{\partial x_{i}^{1}}\right) d s=0 .
$$




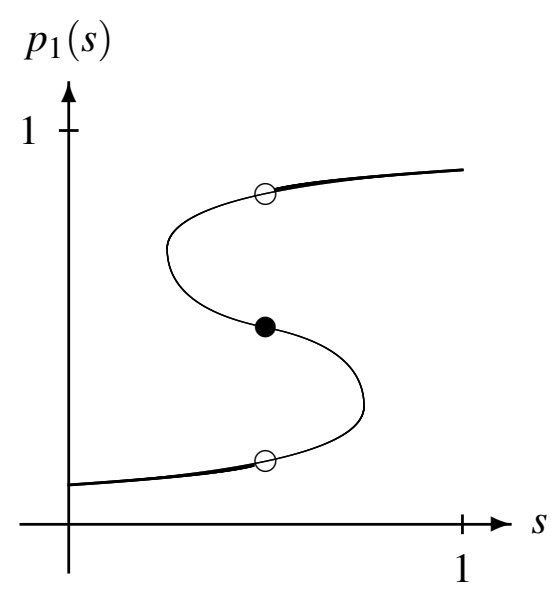

Figure 2: The Walras correspondence $W$ and the selection $r$.

Then $((\bar{p}, \bar{q}),(\bar{x}, \bar{\theta}))$ is a financial market equilibrium and the asset price at date 1 is discontinuous at $s=1 / 2$.

Since $q_{1}(s) \theta_{i}^{0}=\left(x_{i}^{1}(s)-\omega_{i}^{1}(s)\right)+q_{1}(s)\left(x_{i}^{2}(s)-\omega_{i}^{2}(s)\right)$ for both $i$ and almost all $s$, the portfolio $\bar{\theta}_{1}^{0}$ of consumer 1 at date 0 satisfies $\theta_{0}^{1} \in\left[\delta^{L}, \delta^{U}\right]$ for $\delta^{L}=-\inf _{s}\left(\omega_{1}^{1}(s) / q_{1}(s)+\omega_{1}^{2}(s)\right)$ and $\delta^{U}=\inf _{s}\left(\omega_{1}^{1}(s) / q_{1}(s)+\omega_{1}^{2}(s)\right)$. Assume $\omega_{1}^{1}(s), \omega_{1}^{2}(s)<\varepsilon$ for $s \in\{0,1\}$ and the marginal rates of substitution at the Pareto optimal allocations in the sub-economies for $s \in\{0,1\}$ are bounded away from zero and infinity. Then $\lim _{\varepsilon \rightarrow 0} \delta^{L}=\lim _{\varepsilon \rightarrow 0} \delta^{U}=0$. Moreover there is $\bar{\varepsilon}>0$ such that if $\varepsilon \leq \bar{\varepsilon}$, then the set of equilibria for the collection of sub-economies is $S$-shaped for all feasible date 0 portfolios. Therefore $\bar{q}$ is discontinuous in s. Q.E.D.

Remark: The proof of Theorem 4 reveals that any measurable selection $r: S \rightarrow \mathbb{R}_{++}^{2}$ such that $r_{1}(s)=1-r_{1}(1-s)$ and $r_{2}(s)=1-r_{2}(1-s)$ is part of a financial market equilibrium. Therefore as shown in Mas-Colell (1991) there is a continuum of financial market equilibria.

Remark: An crucial difference between the examples in Mas-Colell (1991) and in the proof of Theorem 4 is the number of dates. In Mas-Colell (1991) there are two dates and there is a single asset traded at the first date. Hence the asset price at the first date is unaffected by the shock at the second date so jumps in asset prices are impossible. In the proof of Theorem 4 there are three dates and a single asset traded at the two first dates. Thus the asset price on the second date is affected by the shock at that date making jumps in asset prices possible.

\section{References}

Andersen, T., L. Benzoni \& J. Lund (2002), An empirical investigation of continuous-time equity return models, Journal of Finance 57, 1230-1284. 
Araujo, A., P. Monteiro \& M. Pascoa (1996), Infinite horizon incomplete markets with a continuum of states, Mathematical Finance 6, 119-132.

Balduzzi, P., S. Foresi \& D. Hait (1997), Price barriers and the dynamics of asset prices in equilibrium, Journal of Financial and Quantitative Analysis 32, 137-159.

Bansal, R., \& I. Shaliastovich (2009), Learning and asset price jumps, Review of Financial Studies 24, 2738-2780.

Beker, P., \& S. Chattopadhyay (2010), Consumption dynamics in general equilibrium: A characterisation when markets are incomplete, Journal of Economic Theory 145, 2133-2185.

Calvet, L., (2001), Incomplete markets and volatility, Journal of Economic Theory 98, 295-338.

Calvet, L., \& A. Fischer (2008), Multifrequency jump diffusions: An equilibrium approach, Journal of Mathematical Economics 44, 207-226.

Chichilnisky, G., \& Y. Zhou (1998), Smooth infinite economies, Journal of Mathematical Economics 29, 27-42.

Christoffersen, P., K. Jacobs \& C. Ornthanalai (2011), Dynamic jump intensities and risk premia: evidence from S\&P 500 returns and options, Journal of Financial Economics 106, 447-472.

Citanna, A., \& K. Schmedders (2005), Excess price volatility and financial innovation, Economic Theory 26, 559-588.

Constantinides, G. A., \& D. Duffie (1996), Asset pricing with heterogeneous consumers, Journal of Political Economy 104, 219-240

Covarrubias, E., (2010), Regular infinite economies, B.E. Journal of Theoretical Economics 10, 1-21.

Elul, R., (1999), Effectively complete equilibria - a note, Journal of Mathematical Economics 32, 113-119.

Eraker, B., M. Johannes \& N. Polson (2003), The impact of jumps in volatility and returns, Journal of Finance 58, 1269-1300.

Ghiglino, C., \& M. Tvede (1997), Multiplicity of equilibria, Journal of Economic Theory $75,1-15$.

Heaton, J., \& D. Lucas (1996), Evaluating the effects of incomplete markets on risk sharing and asset pricing, Journal of Political Economy 104, 443-487. 
Kubler, F., \& K. Schmedders (2003), Generic inefficiency of equilibria in the general equilibrium model with incomplete asset markets and infinite time, Economic Theory 22, $1-15$.

Lim, G., V. Martin \& L. Teo (1998), Endogenous jumping and asset price dynamics, Macroeconomic Dynamics 2, 213-237.

Lucas, D., (1994), Asset pricing with undiversifiable income risk and short sales constraints: deepening the equity premium puzzle, Journal of Monetary Economics 34, $325-341$.

Mas-Colell, A., (1991), Indeterminacy in incomplete market economies, Economic Theory 1, 45-61.

Mas-Colell, A., \& W. Zame (1996), The existence of security market equilibrium with a non-atomic state space, Journal of Mathematical Economics 26, 63-84.

Merton, R., (1976), Option pricing when the underlying stock returns are discontinuous, Journal of Financial Economics 3, 125-144.

Radner, R., (1972), Existence of equilibrium in plans, prices, and expectations in a sequence of markets, Econometrica 40, 289-303.

Telmer, C. I. (1993), Asset-pricing puzzles and incomplete markets, Journal of Finance 48, 1803-1832. 